\title{
PLANTAS SILVESTRES ÚTILES Y PRIORITARIAS IDENTIFICADAS EN LA MIXTECA POBLANA, MÉXICO
}

\author{
Aniceto Martínez-Pérez ${ }^{1}$, Pedro Antonio López ${ }^{1,3}$, Abel Gil-Muñoz y y Jesús \\ AxayÁcatl Cuevas-Sánchez ${ }^{2}$ \\ ${ }^{1}$ Colegio de Postgraduados, Campus Puebla, km 125.5 carretera México-Puebla, \\ Santiago Momoxpan, 72760 San Pedro Cholula, Puebla, México. \\ ${ }^{2}$ Universidad Autónoma Chapingo, Departamento de Fitotecnia, km. 38.5 carretera \\ México-Texcoco, 56230 Chapingo, México. \\ 33Autor para la correspondencia: palopez@colpos.mx
}

\section{RESUMEN}

Uno de los factores de la producción sostenible de los bosques tropicales es la conservación de sus especies vegetales silvestres útiles, cuyo estado puede ser evaluado inicialmente a través del conocimiento tradicional local. El objetivo de este trabajo fue contribuir a la elaboración de un inventario de plantas útiles y, mediante valoración socioeconómica y ecológica, determinar prioridades de conservación en la Mixteca Poblana, México. Se realizaron 14 entrevistas y un taller participativo en dos comunidades del municipio de Chietla, Puebla, México. Se registraron los usos de las especies reconocidas, la importancia socioeconómica y el potencial ecológico de cada planta considerada relevante y se aplicó un análisis de factores principales. En ambas comunidades se reconocieron nueve categorías de uso, destacando las combustibles, las medicinales, las alimenticias y las de construcción. En Huajotitlán se reportaron 139 especies útiles, ubicando a palo dulce (Eysenhardtia polystachya), pitaya (Stenocereus stellatus) y palma (Brahea dulcis) como adecuadas para planes de manejo y aprovechamiento; las especies prioritarias para reforestación fueron cuachalalate (Amphipterygium adstringens), cuatomate (Solanum glaucescens), nanche (Byrsonima crassifolia), quina (Hintonia latiflora) y rabo de iguana (Havardia acatlensis). En Buenavista se reportaron 126 especies útiles, de las cuales pitaya y palma fueron percibidas como importantes para su manejo y aprovechamiento; ciruela (Spondias purpurea), palo dulce y árnica (Colubrina triflora) fueron identificadas como apropiadas para reforestación. El análisis de factores principales confirmó la importancia de las especies en cada comunidad. 
Palabras clave: análisis de factores principales, conocimiento tradicional local, conservación, México, plantas útiles, Puebla.

\begin{abstract}
Achieving a sustainable production in tropical forests depends, among other factors, upon the conservation of their useful wild plant species. A key element for such conservation is traditional local knowledge, since it allows a preliminary evaluation of the current situation of those species. With the purpose of contributing to the elaboration of an inventory of useful plants and to define some management and conservation priorities in two communities from la Mixteca Poblana in Mexico, 14 interviews and a participatory workshop were carried out at two communities in the municipality of Chietla, Puebla, Mexico. Uses, socioeconomic importance, and ecological potential for recognized plant species identified as relevant by local people were recorded; a principal factor analysis was carried out. At both communities, nine use categories were recognized; four of them (fuel, medicinal, food, and construction) were the most valued. At Huajotitlán, 139 useful species were reported. Palo dulce (Eysenhardtia polystachya), pitaya (Stenocereus stellatus), and palma (Brahea dulcis), were considered the most relevant for management proposals; cuachalalate (Amphipterygium adstringens), cuatomate (Solanum glaucescens), nanche (Byrsonima crassifolia), quina (Hintonia latiflora), and rabo de iguana (Havardia acatlensis) were priority species for reforestation. At Buenavista, 126 useful species were recognized; of these, only pitaya and palma were considered suitable for management and utilization strategies; ciruela (Spondias purpurea), palo dulce, and arnica (Colubrina triflora) were deemed convenient for reforestation purposes. The principal factor analysis ratified the importance of species at each community.
\end{abstract}

Key words: conservation, Mexico, principal factor analysis, Puebla, traditional local knowledge, useful plants.

\title{
INTRODUCCIÓN
}

Los ecosistemas han sido fuente de bienes y servicios para las sociedades humanas, pero en los últimos años su estructura y funcionamiento han sido afectados (Anónimo, 2005a), originando lo que Dirzo (1990) y Toledo (1994) denominaron respectivamente crisis de la biodiversidad y crisis ecológica, abatiendo los niveles de 
producción de los recursos a tal grado que la supervivencia de las futuras generaciones del hombre está en riesgo (Ehrlich y Ehrlich, 1992; Daily et al., 1999).

Los procesos de destrucción de la vegetación durante las últimas décadas redujeron las áreas boscosas en algunas de las regiones más biodiversas del mundo hasta 25\% o menos de la cubierta original (Aldete et al., 2006). En México, la superficie deforestada llegó a estimarse hasta en 1,500,000 ha anuales (Toledo et al., 1989), aunque recientemente se informó que en los períodos 1990-2000 y 2000-2005 las pérdidas fueron de 631,000 y 260,000 ha al año, respectivamente (Anónimo, 2006).

Una de las primeras y principales acciones a realizar para la conservación consiste en elaborar un inventario y documentar apropiadamente la flora existente en las regiones de interés, a fin de obtener información básica que facilite la preparación de estrategias de conservación y de esta forma establecer mecanismos apropiados para su aprovechamiento racional (Alcorn, 1995; Anónimo, 2000a; Aldete et al., 2006). Para ello, debe tenerse en cuenta que en México gran parte de las especies silvestres útiles se encuentran en zonas de propiedad común, donde la gente local obtiene de forma tradicional diversos productos de su ambiente inmediato, que son útiles para su supervivencia; lo anterior obliga a poner atención a los factores socioeconómicos locales (Anónimo, 1996). Con esta finalidad ha sido ampliamente utilizada la etnobotánica, documentando los recursos vegetales de diversas regiones del mundo, e involucrando a la gente local que se beneficia de ellos. Así, es factible determinar, en forma rápida y eficiente, prioridades de conservación explorando el conocimiento local sobre el estado en que se encuentran las especies de mayor utilidad (Höft et al., 1999; Paule y Potvin, 2004).

Sánchez (1996) documentó la devastación de las poblaciones de copal (Bursera spp.) en comunidades de los valles de Oaxaca; Hellier et al. (1999) estimaron el grado de daño o extinción en poblaciones de especies vegetales y animales en una región montañosa de Chiapas, México; Hersh-Martínez (1995) y Hersh-Martínez y Fierro (2001) percibieron la declinación casi completa de las poblaciones naturales de cancerina (Hemiangium excelsum), quina amarilla (Hintonia latiflora) y quina roja (Simira mexicana) en el extremo suroccidental del estado de Puebla; Caballero y Cortés (2001) encontraron que la palma de guano (Sabal mexicana y S. yapa) casi ha desaparecido del medio natural de la zona maya de Yucatán. Con el propósito de estimular la participación de la gente local en actividades de manejo y conservación, tales esfuerzos tienen que ser orientados hacia los recursos vegetales más importantes para las economías locales (Ticktin et al., 2002). Para saber cuáles son estas plantas promisorias en las comunidades, Arias y Cárdenas 
(2007) sugieren realizar una evaluación de las especies útiles considerando factores sociales, económicos y ecológicos, mediante métodos participativos. Por su parte Höft et al. (1999), Albuquerque et al. (2006), Rivera et al. (2007) y Albuquerque (2009) resaltan la importancia de los estudios cuantitativos en la etnobotánica. Höft et al. (1999) describen la aplicación de algunas técnicas de análisis multivariado como el análisis de conglomerados, de componentes y de factores principales. Respecto al método de análisis de factores principales, el objetivo del mismo es explicar los resultados de un número $p$ de variables originales de una matriz de datos, empleando un menor número de variables, llamadas factores (Anónimo, 2004; Härdle y Simar, 2007).

Para México se tienen registradas 23,424 especies de plantas vasculares, agrupadas en 2804 géneros nativos, comprendidos en 304 familias (Villaseñor, 2004). Además de éstas, se reconocen en el país 618 especies introducidas y naturalizadas (Villaseñor y Espinosa-García, 2004). Esta gran diversidad vegetal se encuentra distribuida en 17 provincias florísticas, 11 de las cuales ocupan la mayor parte del país y están ubicadas en el reino neotropical en el que destaca, por su número importante de endemismos, la Cuenca del Río Balsas, que está ubicada entre el Eje Volcánico Transversal y la Sierra Madre del Sur (Rzedowski, 2006). La vegetación de esta Cuenca ha sufrido procesos tan acelerados de destrucción por actividades de aprovechamiento insostenible, incendios y cambios de uso del suelo principalmente, que en sólo 15 años (entre 1980 y 1996) la extensión forestal se redujo de 960,340 a 291,000 ha (Toledo, 2003).

El Alto Balsas, una de las tres subregiones de esta Cuenca, abarca la Sierra Mixteca Poblana donde se localiza el territorio municipal de Chietla (García-Vázquez et al., 2006) y ha constituido para las comunidades locales una fuente importante de productos vegetales, pero su intensa y desordenada extracción ha ocasionado la pronta disminución de los recursos forestales (Guízar y Sánchez, 1991). Sin embargo, no hay estudios que indiquen cuáles son las especies silvestres útiles e importantes, ni su estado de conservación, que pudieran ser base para el diseño y aplicación de estrategias de conservación. Por ello, es imprescindible documentar en esta zona las plantas actualmente sujetas a aprovechamiento, e indicar cuáles de ellas son las más apreciadas por la población local, a fin de que sean atendidas mediante programas específicos para fortalecer la estabilidad forestal desde la perspectiva local; pues, de acuerdo con Tyler (2006), resguardar y restaurar ecosistemas desde el manejo local beneficia tanto a los usuarios cercanos como a los pueblos de otras regiones, incluso países. En tal contexto, el objetivo de este estudio fue contribuir a la elaboración de un inventario de plantas útiles -primordialmente silvestres- de las áreas cerriles del 
municipio de Chietla, Puebla, y evaluar algunos aspectos socioeconómicos y ecológicos asociados a la importancia atribuida a éstas por parte de habitantes de las comunidades involucradas en el presente estudio.

\section{MATERIALES Y MÉTODOS}

\section{Localización}

El trabajo se realizó en las comunidades de San Lucas Huajotitlán (Huajotitlán) y Buenavista de Juárez (Buenavista), ambas pertenecientes al municipio de Chietla, Puebla (Fig. 1) y localizadas dentro de la región denominada por Fernández et al. (1998) como subprovincia fisiográfica "Sur de Puebla", la cual conforma uno de los sectores limítrofes de la Cuenca del Río Balsas. Las porciones cerriles que les proveen recursos vegetales silvestres son adyacentes y poseen el tipo de vegetación

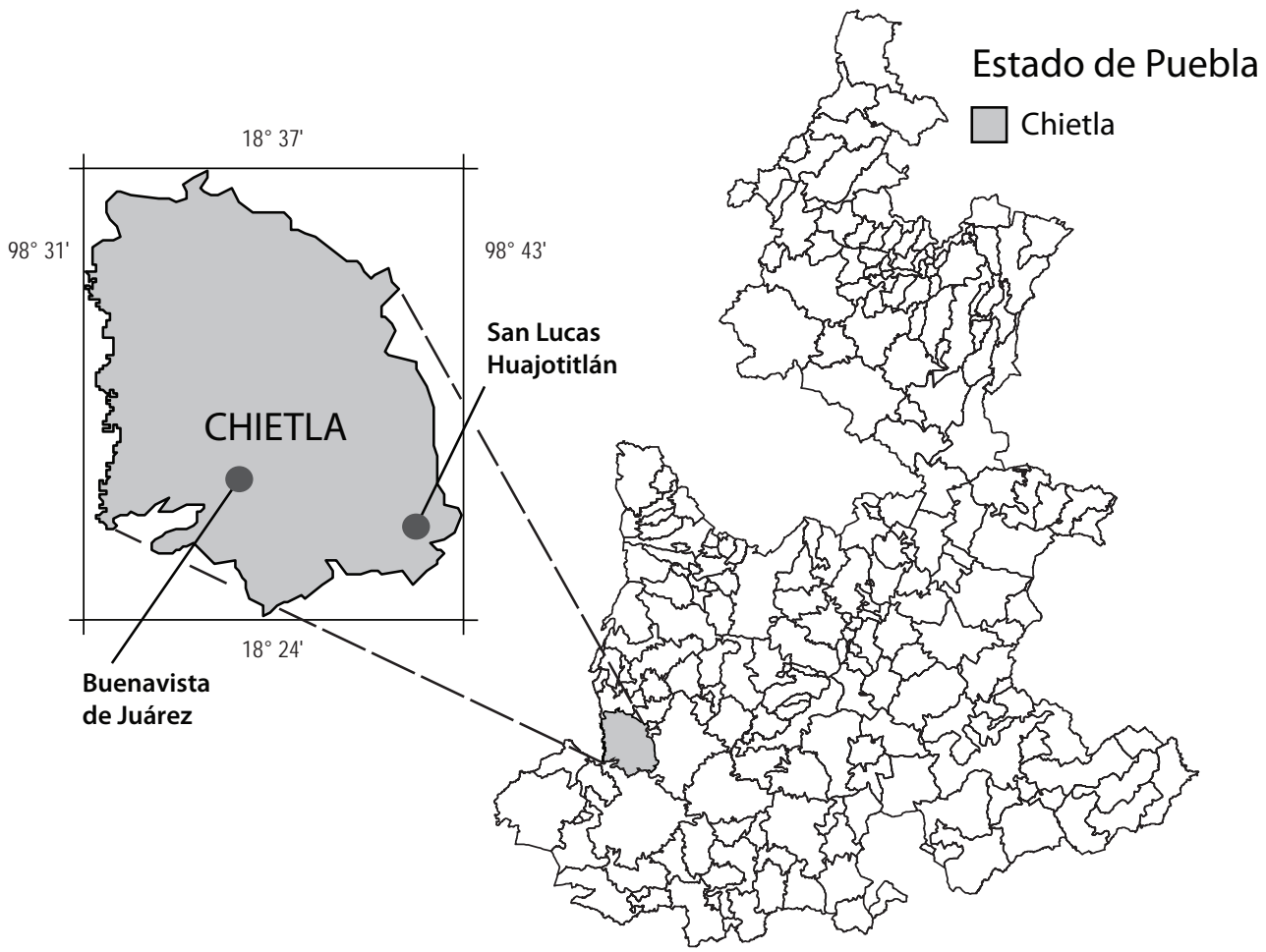

Fig. 1. Ubicación de las comunidades en las cuales se efectuó el estudio. 
reportado como selva baja caducifolia (Miranda y Hernández X., 1963) y descrito por Rzedowski (2006) como bosque tropical caducifolio.

La selva baja caducifolia, de acuerdo con Miranda y Hernández X. (1963) se caracteriza por estar conformada por árboles hasta de $15 \mathrm{~m}$ de altura, que pierden las hojas en época seca y por lo general no son espinosos; se relaciona con climas semisecos o subsecos y cálidos, con temperatura media anual superior a $20{ }^{\circ} \mathrm{C}$, precipitación anual media entre 500 y 1200 mm y estación seca larga y marcada. En el centro de México este tipo de vegetación se encuentra en declives de la cuenca del Balsas y de la cuenca alta del Papaloapan, donde se pueden encontrar especies como los cuajiotes (Bursera spp.), el copaljocote o chupandía (Cyrtocarpa procera), el brasil (Haematoxylon brasiletto), los cazahuates (lpomoea spp.). Las tierras propias de esta clase de selva, cuando son medianamente profundas, son usadas para cultivos trashumantes de maíz y ajonjolí de temporal; la ganadería se aprovecha del ramoneo y de los pastizales inducidos de Cathestecum y Opizia. En el área de estudio abundan los suelos de tipo litosol, caracterizados por ser de poca profundidad, sin horizontes desarrollados, escasa retención de humedad, muy pedregosos y de moderada fertilidad (Anónimo, 2000b).

En las partes más altas del municipio de Chietla, donde se localiza la comunidad de Huajotitlán, el Instituto Nacional de Estadística y Geografía (Anónimo, 2000b) reporta el tipo de clima (A)Cw ${ }_{0}$ w, que según García (1973) es el menos húmedo dentro de los semicálidos subhúmedos, con lluvias en verano, una época seca larga en el invierno y una corta en el verano. La precipitación y temperatura promedio anuales van de 800 a $1000 \mathrm{~mm}$ y de 18 a $22{ }^{\circ} \mathrm{C}$ respectivamente, y la temperatura media del mes más frío es superior a los $18^{\circ} \mathrm{C}$. El tipo de clima presente en la mayor parte del territorio del municipio de Chietla, incluyendo a la comunidad de Buenavista, corresponde al Aw” ${ }_{0}(\mathrm{w})(\mathrm{i}$ )g, el más seco de los cálido subhúmedos, con lluvias en verano, una época seca larga en el invierno y una corta en el verano. Los registros de precipitación y temperatura indican un promedio anual de $806.7 \mathrm{~mm}$ y $24.3^{\circ} \mathrm{C}$ respectivamente, y la temperatura media del mes más frío es de $20.8{ }^{\circ} \mathrm{C}$ (García, 1973).

La comunidad de Huajotitlán está situada en una zona cerril, entre las coordenadas $98^{\circ} 32^{\prime} 27^{\prime \prime} \mathrm{LO}$ y $1^{\circ} 26^{\prime} 08^{\prime \prime} \mathrm{LN}$, a una elevación de 1350 m s.n.m. La población sólo cuenta con 43 habitantes, de los cuales 22 son mayores de 18 años (Anónimo, 2005a). Información proporcionada por Domingo Arrieta (inspector municipal) indica que el poblado carece de todos los servicios básicos y está asentado en terrenos de uso comunal pertenecientes al ejido de Viborillas de Hidalgo. Cada familia dispone de dos a tres hectáreas para el cultivo de maíz, frijol y cacahuate durante la temporada de lluvias, por lo que la agricultura es una de sus principales actividades 
económicas, junto con la elaboración y venta de carbón, así como la colecta y venta de leña, de plantas y frutos comestibles y medicinales.

Buenavista está ubicada en la planicie del municipio de Chietla entre las coordenadas 98³8'11" LO y 18²7'38" LN, a una elevación de 1060 m, su población total es de 1280 habitantes, de los cuales 662 son mayores de edad (Anónimo, 2005a). Según Claudio Romero (presidente del comisariado ejidal), el ejido de Buenavista cuenta con 1297.5 ha, repartidas en 876.36 ha de área cerril comunal, 359.25 ha de uso agrícola (distribuidas entre 84 ejidatarios, con una dotación promedio de 0.77 ha de riego y tres hectáreas de temporal cada uno), y 61.89 ha de asentamiento humano; la población cuenta con todos los servicios básicos, sus principales actividades económicas son: i) la agricultura, en la que sorgo, cebolla y caña de azúcar son los cultivos más importantes, por la superficie sembrada y cuya cosecha es destinada a la venta, mientras que la producción de maíz y cacahuate es dedicada al consumo familiar; ii) la ganadería y iii) el comercio en pequeña escala, así como la colecta de productos vegetales para diversos usos.

Recopilación de la información

La información etnobotánica que se describe más adelante fue recopilada en el período de junio de 2007 a noviembre de 2008. Para colectarla se siguieron las recomendaciones de Martin (1995), Casana-Martínez et al. (1996), Blanco et al. (2000) y Hoffman y Gallaher (2007), en cuanto a que en las dos comunidades se solicitó la colaboración de las personas más destacadas en el conocimiento y uso de la vegetación que crece en las zonas cerriles, logrando conformar así un grupo de siete informantes clave. Éstos fueron seleccionados considerando su disposición a colaborar, detectada durante una entrevista a las personas que sugirieron las autoridades locales. Las técnicas a las cuales se recurrió para obtener la información fueron la aplicación de entrevistas (Martin, 1995; Blanco-Castro, 1996; Casana-Martínez et al. 1996; Hoffman y Gallaher, 2007), recorridos de campo y talleres participativos (Martin, 1995; Arias y Cárdenas, 2007; Hoffman y Gallaher, 2007).

A cada uno de los informantes clave se le aplicó una entrevista semi-estructurada, en la que se solicitaron principalmente sus datos personales (nombre, edad, escolaridad y actividades principales); y los relativos a todas las plantas no cultivadas (nombre local de la planta, forma biológica -árbol, arbusto, hierba, trepadora-, lugar de obtención, época de obtención, partes usadas y todos los tipos de uso) que utilizaba para cualquier finalidad.

Adicionalmente, en compañía de algunos informantes clave, se realizaron siete recorridos de campo por las zonas de recolección más frecuentadas por ellos 
(áreas de acción cotidiana); durante los mismos, y de acuerdo con lo sugerido por Hernández-Xolocotzi (1970), Martin (1995), Alexiades (1996) y Hoffman y Gallaher (2007), se colectaron ejemplares de las diferentes especies útiles localizadas. Éstos fueron herborizados y se enviaron al herbario de la Benemérita Universidad Autónoma de Puebla para su identificación. Un duplicado de 50 especímenes fue enviado al herbario XOLO de la Universidad Autónoma Chapingo para su cotejo taxonómico. Algunas de las especies reconocidas fueron identificadas con base en información consultada en la literatura y otras con ejemplares de herbario.

\section{Análisis de la información}

Definición de categorías de uso. A partir de la información obtenida durante las entrevistas semi-estructuradas, se procedió a agrupar a las especies en categorías de uso, de acuerdo con lo señalado por los entrevistados en: a) combustibles: especies arbóreas o arbustivas cuyos tallos y ramas son utilizadas como leña o para elaborar carbón; b) medicinales: las utilizadas en el tratamiento o prevención de enfermedades; c) alimenticias: las usadas como comestibles; d) forrajeras: las que son alimento para animales domésticos; e) construcción: las explotadas en la edificación de viviendas, cobertizos o cercas, como horcones, vigas, postes, techos, amarres, etc.; f) aparejos: las que sirven para elaborar partes de monturas e implementos agrícolas como timones, yugos, clavijas, manceras, etc.; g) ceremoniales: las aprovechadas para adornar espacios donde se llevan a cabo ceremonias religiosas; h) ornamentales: las empleadas para embellecer espacios urbanos (patios, jardines, plazas, parques, etc.); y i) otras: que incluyen a las que no fueron ubicadas en alguna de las categorías anteriores.

Valoración y selección de especies. Con el fin de llegar a un consenso respecto a cuáles eran las categorías de uso que representaban mayor relevancia para las comunidades, así como las especies de mayor aprecio dentro de éstas, tanto por su valor de uso como por el de cambio, a cada uno de los informantes se le presentó una relación con los nombres de las categorías y plantas registradas en la etapa anterior. Este listado se acompañó de un formato para anotar y valorar a las especies seleccionadas de acuerdo con Peters (1996), incluyó variables de carácter social, económico y ecológico (Cuadro 1). La valoración se efectuó mediante una escala de calificación gráfica, técnica ampliamente aplicada en estudios de psicología y en paneles de evaluación sensorial (Lawless y Heymann, 1998), que consiste en solicitar al evaluador que marque sobre una línea horizontal de longitud conocida dibujada en la hoja de respuestas, la importancia relativa de un producto o atributo; en nuestro caso, a cada especie por categoría se le asoció una línea recta de $10 \mathrm{~cm}$ de longitud (que representaba una escala de calificación para la especie de 0 a 100 puntos en cada variable), a 
Cuadro 1. Variables usadas para evaluar en forma relativa la importancia socioeconómica y factores ecológicos de las especies seleccionadas en San Lucas Huajotitlán y en Buenavista de Juárez, Chietla, Puebla, durante el período julio-diciembre de 2008.

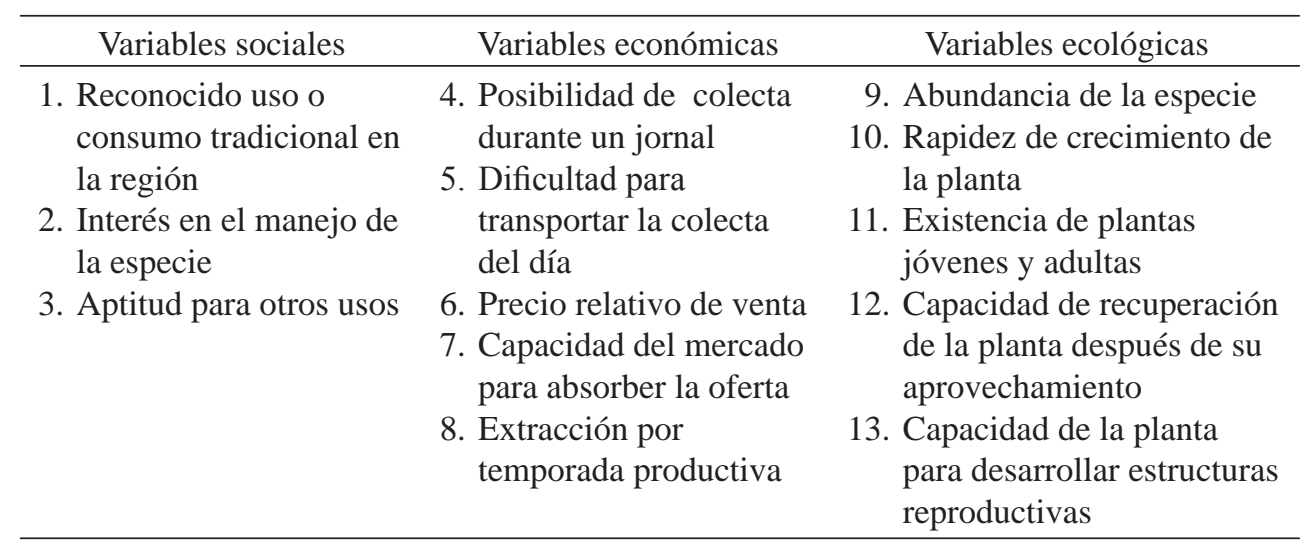

fin de que el informante, después de hacer una comparación mental con las demás entidades de su categoría, calificara la especie en cuestión, colocando para ello una marca sobre la línea en el sitio que consideraba le correspondía.

Las calificaciones asignadas por cada informante para cada planta y variable, se calcularon midiendo en milímetros la longitud del segmento de recta desde 0 hasta la marca hecha por el evaluador. Posteriormente, para cada comunidad, a partir de las calificaciones individuales de cada especie y variable, se calcularon los promedios respectivos. Las plantas que obtuvieron los promedios más altos para una variable determinada fueron consideradas como sobresalientes para dicha característica. Dependiendo del número de variables (sociales, económicas o ecológicas) para la cual resultó sobresaliente una planta dada, se procedió a priorizarlas como se explica a continuación: especies de importancia socioeconómica mayor fueron aquellas que sobresalieron en 2-3 variables sociales y en 3-5 económicas; las de mediana importancia, las que sobresalieron en 1 variable social y en 1-2 económicas; las de relevancia menor, las que no sobresalieron en variable alguna. Especies de mayor abundancia o con mayores posibilidades de incrementar sus poblaciones, las que sobresalieron en 3-5 variables ecológicas; especies de abundancia regular, las que sobresalieron en 1-2 variables ecológicas, y especies escasas o amenazadas, las que no fueron sobresalientes en alguna.

Análisis estadístico. Dado que las variables involucradas fueron numerosas e independientes, con el fin de reducir la dimensionalidad de los datos obtenidos 
de la valoración y facilitar su interpretación, los promedios de estas calificaciones fueron sometidos a un análisis de factores principales exploratorio (Pérez-Gil et al., 2000), dado que no se conocía a priori el número de factores que podrían explicar las interrelaciones entre las variables originales (Sharma, 1996; Höft et al., 1999; Härdle y Simar, 2007), utilizando el programa estadístico SPSS (Statistical Package for the Social Sciences) versión 15.0.1 (Anónimo, 1989). Brevemente, el proceso de análisis estadístico consistió en la elaboración de una matriz de datos con las especies de plantas registradas como de mayor aprecio por los habitantes de las dos comunidades en 53 hileras y las calificaciones asignadas para cada variable en 13 columnas. Los factores principales se extrajeron mediante el método de componentes principales, aplicado a la matriz de correlaciones, seleccionando a los factores con valores propios mayores a la unidad; inicialmente se realizó el análisis factorial sin rotación y posteriormente se aplicó el método de rotación varimax, para maximizar la varianza (Härdle y Simar, 2007); como pruebas de bondad de ajuste del modelo se aplicaron la de Kaiser-Meyer-Olkin y la prueba de Bartlett (Härdle y Simar, 2007).

\section{RESULTADOS Y DISCUSIÓN}

Riqueza etnoflorística

El total de especies vegetales silvestres señaladas como útiles en ambas comunidades fue de 180 , las cuales representan $4.12 \%$ de la diversidad vegetal reportada para la Cuenca del Río Balsas, que según Fernández et al. (1998) es de 4,442 entidades. Esta riqueza de plantas útiles en términos absolutos, resulta ser ligeramente mayor a la comunicada por Paredes-Flores et al. (2007) en su estudio sobre la vegetación natural del municipio de Zapotitlán Salinas (localizado en el Valle de Tehuacán, Puebla), que asciende a 164 especies. No obstante, en términos relativos, el porcentaje calculado para el presente trabajo es menor al de Zapotitlán, pues en el Valle de Tehuacán, Puebla, que es una zona árida, existen alrededor de 2,800 especies de plantas (Dávila et al., 2002), por lo que el porcentaje de especies útiles correspondiente a Zapotitlán Salinas es de 5.85\%; 47.8\% de ellas fueron comunes a las dos comunidades bajo estudio, mientras que 30.0\% fueron exclusivas de Huajotitlán y 22.2\% de Buenavista.

En Huajotitlán se reportaron 139 especies en total, de las cuales 106 estuvieron relacionadas con un uso exclusivo y 36 con varios empleos. Al analizar el número de plantas registradas por categoría de utilidad en esta localidad, se observa que las más abundantes fueron las combustibles, las medicinales y las alimenticias. 
Con menor frecuencia se ubicaron las forrajeras, las utilizadas para construcción y para aparejos. Las menos repetidas resultaron ser las de uso ceremonial, ornamental o con otra aplicación (Cuadro 2). En Buenavista se registraron 126 especies en total, de las cuales 89 fueron reportadas por tener solamente un uso y 38 con más de uno. En esta localidad, la categoría más numerosa fue la de medicinales, seguida por las combustibles, las de construcción y las alimenticias. Con menos frecuencia aparecieron las forrajeras, las empleadas para aparejos, las ceremoniales, ornamentales y las destinadas para otros usos (Cuadro 2).

Estos resultados muestran que la vegetación silvestre provee a estas comunidades principalmente con productos básicos, además, resultó notorio que las categorías con mayor especificidad de uso fueron las medicinales, las alimenticias, las ceremoniales y las ornamentales, pues más de $60 \%$ de las plantas ubicadas en esas categorías fueron empleadas con un solo propósito. En contraste, las plantas combustibles, forrajeras, para construcción y para aparejos resultaron ser multifun-

Cuadro 2. Categorías de uso y número de especies vegetales silvestres útiles en dos comunidades del municipio de Chietla, Puebla.

\begin{tabular}{lrrrrrrrr}
\hline \multirow{2}{*}{ Categorías de uso } & \multicolumn{3}{c}{ San Lucas Huajotitlán } & & \multicolumn{3}{c}{ Buenavista de Juárez } \\
\cline { 2 - 4 } \cline { 7 - 9 } & $\mathrm{A}$ & $\mathrm{B}$ & $\mathrm{C}$ & & $\mathrm{A}$ & $\mathrm{B}$ & $\mathrm{C}$ \\
\hline Combustibles & 63 & 30 & 33 & & 30 & 3 & 27 \\
Medicinales & 45 & 38 & 7 & & 55 & 42 & 13 \\
Alimenticias & 28 & 20 & 8 & & 23 & 17 & 6 \\
Forrajeras & 18 & 3 & 15 & & 16 & 8 & 8 \\
Construcción & 15 & 4 & 11 & & 30 & 10 & 20 \\
Aparejos & 14 & 6 & 8 & & 13 & 2 & 11 \\
Ceremoniales & 4 & 3 & 1 & & 5 & 3 & 2 \\
Ornamentales & 1 & 1 & 0 & & 2 & 2 & 0 \\
Otras & 4 & 1 & 3 & & 4 & 2 & 2 \\
\hline Total & 192 & 106 & 36 & & 178 & 89 & 38 \\
\hline
\end{tabular}

A: Número de especies registradas en cada categoría.

B: Número de especies con sólo el tipo de uso indicado.

C: Número de especies de la categoría indicada, con otros tipos de usos.

Nota: El total indicado en la columna $\mathrm{C}$ no es la suma de las cantidades parciales debido a que las especies consideradas presentaron más de un uso, motivo por el cual tuvieron que contabilizarse varias veces (una por cada categoría de uso en la que hubo de ubicarse). 
cionales en cuanto a que tuvieron cuando menos un uso más, aparte del principal. Hoffman y Gallaher (2007) resaltan que la medición de la importancia de las plantas y la vegetación para la gente local es un enfoque central en la etnobotánica cuantitativa. En otros estudios se ha demostrado el amplio conocimiento que la gente local conserva en relación con las plantas que la rodean (Casas y Caballero, 1996; Albuquerque et al., 2006).

Valoración de especies

Los informantes de ambas comunidades opinaron que la población necesita, utiliza (y en determinadas épocas del año, comercializa) en mayor medida, plantas alimenticias, medicinales y de construcción; en Huajotitlán se agregaron las combustibles. Por tanto, se decidió seleccionar para su valoración las especies que fueron más apreciadas dentro de cada una de estas categorías de uso, y para las cuales los pobladores estimaron conveniente involucrarse en determinadas acciones de manejo.

El Cuadro 3 muestra las especies que los informantes de Huajotitlán decidieron valorar, por ser las de mayor aprovechamiento dentro de las categorías de uso señaladas en el párrafo anterior. Para cada categoría se indica el número de variables sociales, económicas y ecológicas en las cuales resultó sobresaliente una especie. Cabe mencionar que los entrevistados decidieron no incluir las combustibles, debido a que expresaron que de ellas se disponía de una gran variedad y abundancia, por lo que manifestaron no tener interés en involucrarse en eventuales tareas de manejo de las mismas.

Las plantas que resultaron ser de mayor importancia socioeconómica para los habitantes de Huajotitlán fueron cuachalalate (Amphipterygium adstringens), palo dulce (Eysenhardtia polystachya) -en dos categorías de uso-, pitaya (Stenocereus stellatus), cuatomate (Solanum glaucescens) y palma (Brahea dulcis); de éstas, las más abundantes o con mayor capacidad para incrementar sus poblaciones -en la percepción de los agricultores- fueron palo dulce, pitaya y palma, en tanto que cuachalalate fue de abundancia regular y cuatomate resultó ser la más escasa o amenazada. Las especies que reflejaron mediana importancia socioeconómica fueron, zacatechichi (Calea zacatechichi), ciruela (Spondias purpurea), aclina (no identificada), flor de muerto (Galphimia glauca), brasil (Haematoxylum brasiletto), cuatillo, quina (Hintonia latiflora), nanche (Byrsonima crassifolia) y rabo de iguana (Havardia acatlensis); de éstas, las tres primeras se calificaron como de mayor abundancia, las tres siguientes fueron de regular profusión, mientras que las últimas tres resultaron ser las más escasas o con mayores problemas para multiplicarse de manera natural. 
Cuadro 3. Especies más importantes que fueron evaluadas en San Lucas Huajotitlán y número de variables en las que fueron sobresalientes.

\begin{tabular}{|c|c|c|c|}
\hline \multirow{2}{*}{ Especies por categoría de uso } & \multicolumn{3}{|c|}{ Número de variables } \\
\hline & Sociales & Económicas & Ecológicas \\
\hline \multicolumn{4}{|l|}{ Medicinales } \\
\hline $\begin{array}{l}\text { Cuachalalate (Amphipterygium adstringens } \\
\text { Schiede ex Schlecht.) }\end{array}$ & 2 & 4 & 2 \\
\hline $\begin{array}{l}\text { Palo dulce (Eysenhardtia polystachya (Ortega) } \\
\text { Sarg.) }\end{array}$ & 2 & 3 & 3 \\
\hline $\begin{array}{l}\text { Quina (Hintonia latiflora (Sessé \& Moc. ex } \\
\text { DC.) Bullock) }\end{array}$ & 1 & 1 & 0 \\
\hline Zacatechichi (Calea zacatechichi Schltdl.) & 1 & 1 & 5 \\
\hline Flor de muerto (Galphimia glauca Cav.) & 1 & 1 & 1 \\
\hline Palo de tres costillas (Serjania triquetra Radlk.) & 0 & 0 & 1 \\
\hline Margarita ( ) & 0 & 0 & 2 \\
\hline Brasil (Haematoxylum brasiletto H. Karst.) & 2 & 2 & 1 \\
\hline \multicolumn{4}{|l|}{ Alimenticias } \\
\hline Ciruela (Spondias purpurea L.) & 2 & 2 & 4 \\
\hline Pitaya (Stenocereus stellatus (Pfeiff.) Riccob.) & 3 & 4 & 4 \\
\hline Cuatomate (Solanum glaucescens Zucc.) & 2 & 4 & 0 \\
\hline Nanche (Byrsonima crassifolia (L.) Kunth) & 1 & 2 & 0 \\
\hline Guaje (Leucaena spp.) & 1 & 0 & 2 \\
\hline Fraile (Porophyllum spp.) & 0 & 0 & 5 \\
\hline \multicolumn{4}{|l|}{ Construcción } \\
\hline Palma (Brahea dulcis (Kunth) Mart.) & 2 & 4 & 5 \\
\hline Aclina ( ) & 1 & 2 & 5 \\
\hline Cuatillo ( ) & 1 & 1 & 1 \\
\hline $\begin{array}{l}\text { Palo dulce (Eysenhardtia polystachya } \\
\text { (Ortega) Sarg.) }\end{array}$ & 2 & 3 & 4 \\
\hline $\begin{array}{l}\text { Rabo de iguana (Havardia acatlensis } \\
\text { (Benth.) Britton \& Rose) }\end{array}$ & 3 & 2 & 0 \\
\hline
\end{tabular}

( ) Especie no identificada. 
En cuanto a las calificadas como de mínima importancia socioeconómica se tuvieron a palo de tres costillas (Serjania triquetra), margarita (no identificada), guaje (Leucaena spp.) y fraile (Porophyllum spp.), todas con regular a mayor abundancia. Estos resultados muestran que el grupo de plantas con el mayor nivel de valor socioeconómico para los pobladores de Huajotitlán está integrado por cinco especies; encontrándose entre ellas, las que tienen más posibilidades de conservación y recuperación en la percepción de los agricultores, así como algunas que se consideran con mayor necesidad de reforestación. Quina, nanche y rabo de iguana reportaron un menor nivel de importancia socioeconómica, aunque conviene señalar que sus poblaciones fueron de las más escasas, por lo que no se observó una correlación definida entre el nivel de importancia y la abundancia para las plantas registradas.

Los resultados obtenidos en la comunidad de Huajotitlán pueden ser atribuibles a sus mayores restricciones en cuanto a servicios, pues ello obliga a sus habitantes a hacer un aprovechamiento más intensivo de los recursos disponibles en su medio, lo que implica necesariamente el recurrir a un mayor número de especies vegetales. Por otra parte, la ubicación geográfica de esta población (enclavada en una zona cerril) permite a los que ahí habitan una relación muy estrecha con la vegetación circundante, sobre la cual concentran en gran medida sus actividades cotidianas para extraer con relativa frecuencia, materiales vegetales básicos, tanto para autoconsumo como para comercialización, principalmente combustibles, alimenticios y medicinales de alta demanda en la región. Otros autores han demostrado que es con base en el manejo e interacción cotidianos de la gente local con las especies útiles como se prioriza el estado de conservación de las mismas (Pardo y Gómez, 2003; Paule y Potvin, 2004; Paredes-Flores et al., 2007).

El Cuadro 4 conserva la misma estructura del Cuadro 3, pero corresponde a la localidad de Buenavista. En este caso, las especies de mayor importancia socioeconómica fueron pitaya, ciruela, palo dulce (en la categoría de construcción) y palma, entre las cuales pitaya y palma destacaron en las variables ecológicas, indicando así que fueron las más abundantes o las que tienen mejor capacidad para incrementar el tamaño de sus poblaciones (en la percepción de los agricultores). En cambio, ciruela y palo dulce fueron calificadas como plantas de mediana o regular abundancia. Las especies percibidas como de mediana importancia socioeconómica fueron árnica (Colubrina triflora), cuachalalate, palo dulce (en la categoría de medicinales), flor de muerto, nanche, guamúchil (Pithecellobium dulce), cuatomate (Solanum glaucescens), tlahuitole (Lysiloma microphyllum), petlaltía colorada (Comociadia mollisima) y tecolhuixtle (Mimosa benthamii). De éstas, se consideraron de mayor abundancia al cuachalalate, nanche, guamúchil y petlaltía colorada; con poblaciones de mediana 
Cuadro 4. Especies más importantes que fueron evaluadas en Buenavista de Juárez y número de variables en las que fueron sobresalientes.

\begin{tabular}{|c|c|c|c|}
\hline \multirow{2}{*}{ Especies por categoría de uso } & \multicolumn{3}{|c|}{ Número de variables } \\
\hline & Sociales & Económicas & Ecológicas \\
\hline \multicolumn{4}{|l|}{ Medicinales } \\
\hline Árnica (Colubrina triflora Brongn. ex Sweet) & 1 & 2 & 0 \\
\hline $\begin{array}{l}\text { Cuachalalate (Amphipterygium adstringens Schiede } \\
\text { ex Schlecht.) }\end{array}$ & 2 & 2 & 3 \\
\hline Cuatecomate (Crescentia alata Kunth) & 2 & 0 & 1 \\
\hline Tlapanche de venado (Porophyllum sp.) & 0 & 1 & 2 \\
\hline Damiana (Turnera diffusa Willd. ex Schult.) & 0 & 1 & 1 \\
\hline Palo dulce (Eysenhardtia polystachya (Ortega) Sarg.) & 1 & 1 & 0 \\
\hline Paraca (Senna skinneri (Benth.) H.S. Irwin \& Barneby) & 1 & 0 & 1 \\
\hline $\begin{array}{l}\text { Quina (Hintonia latiflora (Sessé \& Moc. ex DC.) } \\
\text { Bullock) }\end{array}$ & 1 & 0 & 1 \\
\hline Zacatechichi (Calea zacatechichi Schltdl.) & 0 & 0 & 5 \\
\hline Flor de muerto (Galphimia glauca Cav.) & 1 & 2 & 2 \\
\hline \multicolumn{4}{|l|}{ Alimenticias } \\
\hline Pitaya (Stenocereus stellatus (Pfeiff.) Riccob.) & 2 & 4 & 4 \\
\hline Ciruela (Spondias purpurea L.) & 2 & 3 & 1 \\
\hline Fraile (Porophyllum sp.) & 0 & 0 & 1 \\
\hline Nanche (Byrsonima crassifolia (L.) Kunth) & 1 & 1 & 3 \\
\hline Guamúchil (Pithecellobium dulce (Roxb.) Benth.) & 2 & 2 & 4 \\
\hline Cuatomate (Solanum glaucescens Zucc.) & 1 & 2 & 2 \\
\hline Huajocote ( ) & 1 & 0 & 0 \\
\hline \multicolumn{4}{|l|}{ Construcción } \\
\hline Tlahuitole (Lysiloma microphyllum Benth.) & 2 & 2 & 2 \\
\hline Tepeguaje (Lysiloma acapulcense (Kunth) Benth.) & 0 & 0 & 4 \\
\hline Petlaltia colorada (Comocladia mollissima Kunth) & 3 & 2 & 3 \\
\hline Tecolhuixtle (Mimosa benthamii J.F. Macbr.) & 1 & 2 & 1 \\
\hline Palo dulce (Eysenhardtia polistachya (Ortega) Sarg.) & 3 & 3 & 1 \\
\hline Mora ( ) & 0 & 0 & 2 \\
\hline Palma (Brahea dulcis (Kunth) Mart.) & 2 & 3 & 4 \\
\hline
\end{tabular}

( ) Especie no identificada. 
profusión, flor de muerto, cuatomate, tlahuitole y tecolhuixtle; árnica y palo dulce fueron percibidas como escasas o amenazadas. Las especies que se consideraron como de mínima importancia socioeconómica fueron zacatechichi, tepeguaje (Lysiloma acapulcense), cuatecomate (Crescentia alata), tlapanche de venado (Porophyllum spp.), damiana (Turnera diffusa), paraca (Senna skinneri), quina, fraile, mora (no identificada) y cuajiote (no identificada); de ellas, las dos primeras fueron las de mayor abundancia, la última resultó ser la más escasa y las restantes se valoraron como poblaciones de mediana profusión.

En Buenavista únicamente pitaya y palma son percibidas por los agricultores como de mayor importancia social y económica y presentan las mejores condiciones ecológicas para incrementar sus poblaciones mediante manejo. En esta comunidad los materiales para la construcción de origen vegetal son empleados mayormente para la construcción de cobertizos para animales domésticos; dado que la durabilidad y resistencia no son aspectos del todo críticos para este tipo de instalaciones, se recurre al empleo de cualquier planta que pueda adaptarse para tales fines, lo cual amplía el abanico de especies vegetales útiles. En contraste, en Huajotitlán, las destinadas a la construcción se ocupan para la edificación de viviendas, por lo que sólo aquellas que aseguren larga duración y alta calidad pueden ser empleadas para dicho propósito. Ello ocasiona que la gama de especies a las que se puede recurrir en Huajotitlán para este uso sea menor. El hecho de que en Huajotitlán se haya reportado mayor cantidad de plantas combustibles que en Buenavista puede explicarse por la inexistencia de red eléctrica y de servicio de repartición de gas LP en la primera población. Ello hace que los habitantes tengan que recurrir a fuentes de combustible alternativas, lo que implica la necesidad de explorar un mayor número de especies y de emplear una mayor cantidad de éstas con fines de generación de energía. Además, como ya se mencionó en párrafos anteriores, los habitantes de esta comunidad comercializan combustible en forma de leña o carbón. Estos resultados confirman lo establecido por Cuevas (1988); Hernández et al. (1991) y por Luna et al. (2003) entre otros, en el sentido de que los factores socioeconómicos son determinantes en el aprovechamiento de la vegetación.

Las categorías identificadas por los pobladores de las dos comunidades de estudio, así como las especies por ellos reconocidas muestran que la diversidad vegetal silvestre de las poblaciones estudiadas es, primordialmente, fuente de productos combustibles, medicinales, alimenticios y de construcción para los pobladores. Estos resultados fortalecen lo estipulado por Cuevas (1988) y González y López (1991), quienes mencionan a las especies combustibles, las medicinales y las alimenticias, junto con las forrajeras, como prioritarias en la investigación etnobotánica en 
México. Asimismo, coinciden con lo declarado por Hernández et al. (1991) para las plantas útiles de Tamaulipas y por Luna et al. (2003) para el caso de los encinos, en el sentido de que no es la diversidad, sino los factores socioeconómicos y culturales los que determinan la prioridad en el uso de las plantas.

La razón del mayor grado de aprecio de las plantas medicinales, alimenticias y para construcción en ambas comunidades puede deberse a que estos tipos de vegetales constituyen una fuente natural de productos de primera necesidad. Ello genera a su vez una demanda tal que es capaz de consumir cualquier cantidad de estos recursos como sea posible recolectar en esa región. Esta condición seguramente contribuyó en gran medida para que los agricultores manifestaran interés por participar en programas de manejo de tales especies, con la esperanza de lograr mayores beneficios.

Según Ticktin et al. (2002), la mayor importancia socioeconómica atribuida localmente a una planta es una condición deseable para impulsar programas de aprovechamiento y conservación en las comunidades; por lo anterior, podemos señalar que en ambas poblaciones donde se desarrolló el estudio es posible establecer prioridades para las estrategias de conservación y aprovechamiento sostenido de la vegetación local, con base en el conocimiento e importancia que la gente local otorga a las especies, de acuerdo con la satisfacción que de ellas reciben hacia sus necesidades más inmediatas, tal como lo han establecido Cuevas (1988), Hernández et al. (1991), Höft et al. (1999), Luna et al. (2003) y Rivera et al. (2007).

\section{Análisis factorial}

El análisis factorial generó la lista de valores propios indicados en el Cuadro 5. A fin de obtener factores y una solución más fáciles de explicar, se procedió a ejecutar una rotación. Los resultados de la misma también se reproducen en el Cuadro 5. Se observa que al extraer los tres factores con valores propios mayores que 1 el porcentaje de varianza explicada de los datos originales fue de $70.9 \%$, lo cual se consideró adecuado, desde el punto de vista de aplicación de la técnica.

El Cuadro 6 muestra las correlaciones entre las variables originales o "saturaciones", y los tres factores seleccionados del Cuadro 5. Analizando las saturaciones relativas de cada variable para cada factor se puede apreciar que el primero (F1) se saturó por las siguientes variables: interés en el manejo de la especie, posibilidad de colecta durante un jornal, dificultad para transportar la colecta de un jornal, extracción por temporada productiva, abundancia de la especie, existencia de plantas jóvenes y adultas, capacidad de la planta para producir estructuras reproductivas y capacidad de recuperación de la planta después de su aprovechamiento. El segundo 
Cuadro 5. Valores propios de los factores y cantidad de varianza explicada por cada uno de ellos.

\begin{tabular}{|c|c|c|c|c|c|c|}
\hline \multirow[t]{2}{*}{ Factor } & \multicolumn{3}{|c|}{ Autovalores iniciales } & \multicolumn{3}{|c|}{$\begin{array}{c}\text { Suma de las saturaciones al cuadrado } \\
\text { de la rotación }\end{array}$} \\
\hline & Total & \% de la varianza & \% acumulado & Total & \% de la varianza & $\%$ acumulado \\
\hline 1 & 5.657 & 43.519 & 43.519 & 5.182 & 39.862 & 39.862 \\
\hline 2 & 1.962 & 15.092 & 58.610 & 2.165 & 16.652 & 56.514 \\
\hline 3 & 1.598 & 12.294 & 70.905 & 1.871 & 14.391 & 70.905 \\
\hline 4 & .854 & 6.572 & 77.477 & & & \\
\hline 5 & .768 & 5.911 & 83.388 & & & \\
\hline 6 & .673 & 5.179 & 88.567 & & & \\
\hline 7 & .389 & 2.993 & 91.560 & & & \\
\hline 8 & .318 & 2.449 & 94.009 & & & \\
\hline 9 & .248 & 1.907 & 95.916 & & & \\
\hline 10 & .202 & 1.554 & 97.470 & & & \\
\hline 11 & .161 & 1.239 & 98.710 & & & \\
\hline 12 & .112 & .864 & 99.574 & & & \\
\hline 13 & .055 & .426 & 100.000 & & & \\
\hline
\end{tabular}

(F2) quedó determinado por las variables reconocido uso o consumo tradicional en la región, precio relativo de venta, y capacidad del mercado para absorber la oferta actual. Por último, en el tercero (F3) intervienen las variables rapidez de crecimiento y aptitud para otros usos. De este modo, F1 reflejó lo que se denominó "aptitud para el manejo de la especie", F2 quedó integrado por lo que se designó "potencial de comercialización de la especie" y F3 por "versatilidad-precocidad de la especie".

El Cuadro 7 muestra los coeficientes obtenidos por cada especie sobresaliente para cada uno de los tres factores ya descritos. En Huajotitlán las plantas que tuvieron los mejores resultados dentro de cada categoría de uso en el factor "aptitud para el manejo" fueron zacatechichi, palo dulce, pitaya, ciruela, fraile, palma, aclina y solerillo. De éstas, pitaya, palma y palo dulce (en la categoría de construcción) resultaron tener el mejor "potencial de comercialización"; pero sólo palo dulce (en la categoría de medicinales) fue relevante en "versatilidad-precocidad". Otras especies que sobresalieron por su alto "potencial de comercialización" y por tener cierta "aptitud para el manejo" fueron cuachalalate, flor de muerto, cuatomate y nanche, aunque 
Cuadro 6. Matriz de estructura factorial (componentes rotados)a .

\begin{tabular}{lccc}
\hline \multirow{2}{*}{ Variables } & \multicolumn{3}{c}{ Factores } \\
\cline { 2 - 4 } & F1 & F2 & F3 \\
\hline Reconocido uso o consumo tradicional en la región & .516 & .689 & .043 \\
Interés en el manejo de la especie & .595 & .589 & .277 \\
Posibilidad de colecta durante un jornal & .853 & .177 & .224 \\
Dificultad para transportar la colecta de un jornal & .664 & .043 & .565 \\
Precio relativo de venta & -.025 & .710 & .088 \\
Capacidad del mercado para absorber la oferta actual & -.125 & .772 & -.275 \\
Extracción por temporada productiva & .749 & .179 & .422 \\
Rapidez de crecimiento de la planta & .305 & -.027 & -.841 \\
Abundancia de la especie & .896 & -.143 & -.106 \\
Existencia de plantas jóvenes y adultas & .871 & -.191 & -.039 \\
Capacidad de la planta para producir estructuras & .843 & .166 & .139 \\
reproductivas & & & \\
Capacidad de recuperación de la planta después de su & .586 & .289 & -.162 \\
aprovechamiento & & & \\
Aptitud para otros usos & .328 & -.100 & .629 \\
\hline
\end{tabular}

a: La rotación ha convergido en 5 iteraciones.

presentaron valores bajos para "versatilidad-precocidad". En este último factor destacaron, además de palo dulce, cuachalalate, brasil, quina y paraca. Los resultados anteriores indican que en Huajotitlán, las especies más susceptibles de ser manejadas con fines comerciales, aprovechando racionalmente su abundancia, son: pitaya, palma y palo dulce; mientras que las más indicadas para implementar programas de reforestación son cuachalalate, flor de muerto, cuatomate y nanche.

En Buenavista, sobresalieron por su alta "aptitud para el manejo" guamúchil, pitaya y palma, pero en su "potencial de comercialización" sólo la pitaya fue relevante y no hubo especie sobresaliente en "versatilidad-precocidad". Por otra parte, cuachalalate, flor de muerto, árnica, cuatecomate, ciruela, nanche y cuatomate, mostraron mayor "potencial de comercialización"; pero ninguna figuró en "aptitud para el manejo"; respecto a la "versatilidad-precocidad", sólo destacaron cuachalalate y cuatecomate. Otras especies con altos valores para este último factor fueron palo dulce, tlahuitole y tecolhuixtle. Para Buenavista, todas las especies con alto "poten- 
Cuadro 7. Coeficientes de tres factores obtenidos mediante análisis factorial para las especies evaluadas en las comunidades de estudio.

\begin{tabular}{|c|c|c|c|c|c|c|c|}
\hline \multicolumn{4}{|c|}{ San Lucas Huajotitlán } & \multicolumn{4}{|c|}{ Buenavista de Juárez } \\
\hline $\begin{array}{l}\text { Especies por categoría } \\
\text { de uso }\end{array}$ & $\mathrm{F} 1$ & F2 & F3 & $\begin{array}{l}\text { Especies por categoría } \\
\text { de uso }\end{array}$ & $\mathrm{F} 1$ & $\mathrm{~F} 2$ & F3 \\
\hline Medicinales & & & & Medicinales & & & \\
\hline Zacatechichi & 1.64 & -0.25 & -2.16 & Cuachalalate & -0.04 & 1.34 & 0.39 \\
\hline Palo dulce & 0.95 & -0.65 & 1.60 & Zacatechichi & -0.04 & -1.15 & -2.08 \\
\hline Cuachalalate & 0.46 & 1.31 & 1.19 & Damiana & -0.49 & -0.60 & -1.13 \\
\hline Brasil & 0.13 & -0.60 & 1.55 & Quina & -0.57 & 0.04 & -0.06 \\
\hline Quina & 0.00 & -0.36 & 1.58 & Flor de muerto & -0.63 & 1.69 & -2.29 \\
\hline Paraca & -0.05 & -0.28 & 1.34 & Paraca & -0.83 & 0.25 & -0.28 \\
\hline Palo de tres costillas & -0.18 & -1.27 & 0.78 & Árnica & -1.06 & 1.35 & -0.65 \\
\hline Flor de muerto & -0.20 & 0.33 & 0.48 & Cuatecomate & -1.21 & 1.36 & 1.00 \\
\hline \multirow[t]{2}{*}{ Margarita } & -0.28 & -1.36 & 0.58 & Tlapanche de venado & -1.24 & -0.72 & -1.79 \\
\hline & & & & Palo dulce & -1.61 & -0.37 & 0.86 \\
\hline Alimenticias & & & & Alimenticias & & & \\
\hline Pitaya & 1.46 & 0.26 & -0.15 & Guamúchil & 0.88 & -0.32 & -0.97 \\
\hline Ciruela & 1.08 & -0.06 & 0.00 & Pitaya & 0.53 & 1.42 & 0.09 \\
\hline Fraile & 1.08 & -0.19 & -1.39 & Ciruela & -0.83 & 1.73 & -0.07 \\
\hline Guaje & 0.32 & -0.49 & -0.06 & Nanche & -0.88 & 1.20 & -0.14 \\
\hline Cuatomate & 0.30 & 0.89 & 0.06 & Huajocote & -1.28 & -1.27 & -0.08 \\
\hline Nanche & 0.04 & 0.53 & 0.57 & Fraile & -1.44 & 0.58 & -1.83 \\
\hline \multirow[t]{2}{*}{ Huajocote } & -0.35 & -0.65 & 0.09 & Cuatomate & -1.88 & 1.63 & -0.73 \\
\hline & & & & Cuahuayote & -2.24 & 0.41 & -0.48 \\
\hline Construcción & & & & Construcción & & & \\
\hline Palma & 1.89 & 0.81 & -0.80 & Palma & 0.94 & -0.55 & -1.60 \\
\hline Aclina & 1.80 & -0.39 & -1.15 & Tlahuitole & -0.13 & -1.07 & 0.32 \\
\hline Solerillo & 1.16 & -0.19 & 0.13 & Petlaltía colorada & -0.54 & -0.89 & 0.20 \\
\hline Palo dulce & 0.89 & 0.18 & 0.29 & Tepeguaje & -0.62 & -2.89 & -0.11 \\
\hline Rabo de iguana & 0.51 & 0.08 & 0.75 & Mora & -0.88 & -2.44 & -0.12 \\
\hline \multirow[t]{2}{*}{ Cuatillo } & 0.37 & -0.11 & 0.44 & Tecolhuixtle & -0.91 & -1.48 & 0.31 \\
\hline & & & & Palo dulce & -1.17 & 0.55 & 1.32 \\
\hline
\end{tabular}

F1: aptitud para el manejo de la especie. F2: potencial de comercialización. F3: versatilidadprecocidad 
cial de comercialización", excepto pitaya, presentaron baja "aptitud para su manejo", lo que significa la necesidad apremiante de reforestar para evitar que esas plantas valiosas desaparezcan de su hábitat.

El análisis factorial en Huajotitlán permitió corroborar lo declarado por los agricultores en el sentido de que, según los coeficientes de los factores "aptitud para el manejo de la especie" y "potencial de comercialización de la especie", pitaya, palma y palo dulce son las plantas que mostraron mejores perspectivas para proponer programas de manejo. No obstante, sólo palo dulce sobresalió en "versatilidad-precocidad"; lo cual indica que tiene más tipos de usos que las otras dos. Adicionalmente, este análisis mostró que zacatechichi, ciruela, fraile, aclina y solerillo poseen las condiciones que les proporcionan buena "aptitud para el manejo", pero les falta "potencial de comercialización" para que puedan ser consideradas como prioritarias en esta comunidad. En lo que respecta a cuachalalate, flor de muerto, árnica, cuatecomate, ciruela, nanche y cuatomate, éstas mostraron alto "potencial comercial" y la mínima "aptitud para el manejo", por lo que se requieren acciones urgentes de reforestación hacia estas plantas.

En Buenavista, las especies con alto "potencial de comercialización" presentaron baja "aptitud para el manejo"; la excepción fue la pitaya, que mostró ambas, lo que permitió ratificar a esta planta como la de mayor prioridad para el manejo en esta comunidad, resaltando a la vez la necesidad apremiante de reforestar las otras especies mencionadas con alto "potencial de comercialización", con el fin de reducir los riesgos inherentes a la sobre recolección que implica esta condición.

Un último aspecto que también conviene resaltar es la coincidencia de resultados en cuanto a la identificación de especies vegetales de mayor interés para los agricultores de ambas comunidades a través de técnicas distintas, pues tanto la valoración efectuada por los informantes como el análisis factorial de los datos identificaron, con un alto grado de concordancia, a las mismas plantas clasificadas como más relevantes. Ello permite por tanto, ratificar la validez del conocimiento tradicional (Pardo y Gómez, 2003) en torno al uso y manejo de recursos vegetales, y valorar la necesidad de continuar con los estudios encaminados a recuperar y sistematizar tal acervo de información para emplearlo, entre otros aspectos, en acciones que beneficien a la misma comunidad, como puede ser la conservación de especies silvestres útiles para la población (Höft et al., 1999; Rivera et al., 2007; Albuquerque et al., 2006; Hoffman y Gallaher, 2007; Albuquerque, 2009). Por otro lado, la aplicación de una técnica de análisis multivariado como el análisis de factores principales demostró ser una herramienta útil para aplicarse en estudios etnobotánicos, tal y como lo proponen diferentes investigadores (Sharma, 1996; Höft et al. 1999; Härdle y Simar, 2007; Albuquerque, 2009). 


\section{COMENTARIOS FINALES}

Con este estudio se ha avanzado hacia el conocimiento de las especies vegetales silvestres útiles de mayor prioridad para dos comunidades en particular, en el municipio de Chietla, en la Mixteca Baja Poblana; no obstante, será conveniente corroborar en campo su relevancia a través de mediciones concretas, principalmente para calcular la oferta natural actual de los productos que pueden ser derivados de ellas.

Se espera que la información originada por este estudio pueda ser de utilidad para orientar la toma de decisiones de la gente local y de aquellos que tienen la responsabilidad de formular y establecer estrategias de restauración, de manejo y de conservación de los recursos naturales en la región.

La conservación de la productividad de la selva baja caducifolia puede promoverse a través de la participación de la gente local en el ámbito cotidiano de acción, si se toma en cuenta el conocimiento e interés que se desarrolla en cada comunidad acerca de las especies que les representan beneficios significativos.

\section{AGRADECIMIENTOS}

Se agradece a las personas de las comunidades de Huajotitlán y Buenavista, Pue., que participaron en la presente investigación, por compartir sus conocimientos. El primer autor agradece a la Coordinación Sectorial de Desarrollo Académico el financiamiento otorgado para la realización de este trabajo; a la Dirección General de Educación Tecnológica Agropecuaria por la Beca-comisión otorgada; a la Subdirección de Enlace Operativo de la Dirección General de Estudios Técnico Agropecuarios (DGETA-Puebla), particularmente al Ing. Héctor René Becerril Toral, por su apoyo a la realización del postgrado; y al Dr. Higinio López Sánchez por sus críticas al manuscrito.

\section{LITERATURA CITADA}

Albuquerque, U. P. 2009. Quantitative ethnobotany or quantification in ethnobotany? Ethnobot. Res. \& Appl. 7: 1-3.

Albuquerque, U. P., R. F. P. Lucena, J. M. Monteiro, A. T. N. Florentino y C. de F. C. B. R. Almeida. 2006. Evaluating two quantitative ethnobotanical techniques. Ethnobot. Res. \& Appl. 4: 51-60. 
Alcorn, J. B. 1995. The scope and aims of ethnobotany in a developing world. In: Schultes, R. E. \& S. Von Reis (eds.). Ethnobotany: Evolution of a discipline. Dioscorides Press. Portland, USA. pp. 23-39.

Aldete, J. M. F., M. Magali y C. Candeira. 2006. El estado del arte de los recursos genéticos en las Américas: conservación, caracterización y utilización. Foro de las Américas para la Investigación y el Desarrollo Tecnológico Agropecuario. Brasilia, DF, Brasil. http://www.iica.int/foragro/cd_prior/Docs/RecFitog.pdf. Consultada el 26 de febrero de 2008.

Alexiades, M. N. 1996. Standard techniques for collecting and preparing herbarium specimens. In: Alexiades, M. N. (ed.). Selected guidelines for ethnobotanical research: A field manual. Advances in Economic Botany 10. The New York Botanical Garden. Bronx, New York, USA. 326 pp.

Anónimo. 1989. Statistical package for the social sciences, versión 15.0.1.1989. Disponible en: http://www.spss/devcentral/.

Anónimo. 1996. Informe sobre el estado de los recursos fitogenéticos en el mundo. Preparado para la Conferencia Técnica Internacional sobre los Recursos Fitogenéticos. Organización de las Naciones Unidas para la Alimentación y la Agricultura. Leipzig, Alemania. 85 pp.

Anónimo. 2000a. Recursos fitogenéticos, compromiso internacional y plan de acción de Leipzig. 26a Conferencia regional de la Food and Agriculture Organization of the United Nations para América Latina y el Caribe. Mérida, México. 10 pp.

Anónimo. 2000b. Síntesis geográfica del estado de Puebla. Instituto Nacional de Estadística Geografía e Informática. Aguascalientes, México. 124 pp.

Anónimo. 2004. SAS Institute Inc. SAS/STAT® 9.1 User’s Guide. Cary, NC: SAS Institute Inc. $5121 \mathrm{pp}$.

Anónimo. 2005a. Ecosystems and human well-being: synthesis. Millenium Ecosystem Assessment. Island Press. Washington, D.C., USA. 85 pp.

Anónimo. 2005b. Conteo de población y vivienda 2005; principales resultados por localidad (ITER)-descarga. Puebla. Instituto Nacional de Estadística, Geografía e Informática. http://www.inegi.org.mx/sistemas/consulta_resultados/default.aspx?c=10395\&s=est. Consultada el 21 de diciembre de 2008.

Anónimo. 2006. La gestión ambiental en México. Secretaría del Medio Ambiente y Recursos Naturales. México, D.F., México. 468 pp.

Arias, G. J. C. y D. Cárdenas. 2007. Manual de identificación, selección y evaluación de oferta de productos forestales no maderables. Instituto Amazónico de Investigaciones Científicas-Sinchi. Bogotá, Colombia. 32 pp.

Blanco-Castro, E. 1996. Ideas metodológicas relativas al trabajo de campo etnobotánico. Monograf. Jard. Bot. Córdoba 3: 89-91.

Blanco, E., C. Cuadrado y R. Morales. 2000. Plantas en la cultura material de Fuenlabrada de los Montes (Extremadura, España). Anales Jard. Bot. Madrid 58(1): 145-162.

Caballero, J. y L. Cortés. 2001. Percepción, uso y manejo tradicional de los recursos vegetales en México. In: Rendón, B., S. Rebollar, J. Caballero y M. A. Martínez (eds.). Plantas, cultura y sociedad. Estudio sobre la relación entre seres humanos y plantas en los albores del siglo XXI. Universidad Autónoma Metropolitana, Unidad Iztapalapa y Secretaría del Medio Ambiente, Recursos Naturales y Pesca. México, D.F., México. pp. 79-100. 
Casana-Martínez, E., R. Galán-Soldevilla y J. E. Hernández-Bermejo. 1996. Registro de datos: preparación y estrategia del trabajo de campo. Monograf. Jard. Bot. Córdoba 3: 57-62.

Casas A. y J. Caballero. 1996. Traditional management and morphological variation in Leucaena esculenta (Fabaceae: Mimosoideae) in the Mixtec region of Guerrero, Mexico. Econ. Bot. 50(2): 167-181.

Cuevas S., J. A. 1988. Recursos fitogenéticos: bases conceptuales para su estudio y conservación. Departamento de Fitotecnia, Universidad Autónoma Chapingo. Chapingo, México. 464 pp.

Cuevas S., J. A., E. Hernández, T. Rojas y J. García. 1991. Estudio de los recursos fitogenéticos en el Totonacapan. In: Ortega P., R., H. G. Palomino, G. Castillo, H. V. González y M. M. Livera. (eds.). Avances en el estudio de los recursos fitogenéticos de México. Sociedad Mexicana de Fitogenética, A.C. Chapingo, México. pp. 137-158.

Daily, G. C., S. Alexander, P. R. Ehrlich, L. Goulder, J. Lubchenco, P. A. Matson, H. A. Mooney, S. Postel, S. H. Schneider, D. Tilman y G. M. Woodwell. 1999. Ecosystem services: benefits supplied to human societies by natural ecosystems. Issues in Ecology 2: 18 pp.

Dávila, P., M. C. Arizmendi, A. Valiente-Banuet, J. L. Villaseñor, A. Casas y R. Lira. 2002. Biological diversity in the Tehuacán-Cuicatlán Valley, México. Biodivers. Conserv. 11: 421-442.

Dirzo, R. 1990. La biodiversidad como crisis ecológica actual ¿Qué sabemos? Ciencias 4: 48-55.

Ehrlich, A. H. y P. R. Ehrlich. 1992. Causes and consequences of the disappearance of biodiversity. In: Sarukhán, J. y R. Dirzo (comps.). México ante los retos de la biodiversidad. Comisión Nacional para el Conocimiento y Uso de la Biodiversidad. México, D.F., México. pp. 43-55.

Fernández, R., C. Rodríguez, M. L. Arreguín, A. Rodríguez. 1998. Listado florístico de la cuenca del río Balsas, México. Polibotánica 9: 1-151.

García, E. 1973. Modificaciones al sistema de clasificación climática de Köeppen para adaptarlo a las condiciones de la República Mexicana. Instituto de Geografía, Universidad Nacional Autónoma de México. México, D.F., México. 246 pp.

García-Vázquez, U., L. Canseco-Márquez, J. L. Aguilar-López, C. A. Hernández-Jiménez, J. Maceda-Cruz, M. G. Gutiérrez-Mayén y E. Y. Melgarejo-Vélez. 2006. Análisis de la distribución de la herpetofauna en la región Mixteca de Puebla, México. In: Ramírez-Bautista, A., L. Canseco-Márquez y F. Mendoza-Quijano (eds.). Inventarios herpetofaunísticos de México: avances en el conocimiento de su biodiversidad. Sociedad Herpetológica Mexicana. Publicaciones Especiales 3. S y G Editores. México, D.F., México. pp. 152-169.

González, L. J. y D. López. 1991. Los recursos vegetales silvestres en el municipio de Texcoco, México. In: Ortega P., R., H. G. Palomino, G. F. Castillo, H. V. González y M. M. Livera (eds.). Avances en el estudio de los recursos fitogenéticos de México. Sociedad Mexicana de Fitogenética, A.C. Chapingo, México. pp. 111-135.

Guízar N., E. y V. A. Sánchez. 1991. Guía para el reconocimiento de los principales árboles del alto Balsas. Universidad Autónoma Chapingo. Chapingo, México. 207 pp. 
Härdle W. y L. Simar. 2007. Applied multivariate statistical analysis. Springer-Verlag. Berlin, Alemania. $458 \mathrm{pp}$.

Hellier, A., A. C. Newton y S. Ochoa. 1999. Use of indigenous knowledge for rapidly assessing trends in biodiversity: a case study from Chiapas, Mexico. Biodivers. Conserv. 8: 869-889.

Hersch-Martinez, P. 1995. Commercialization of wild medicinal plants from southwest Puebla, México. Econ. Bot. 49(2): 197-206.

Hersh-Martínez, P. y A. Fierro. 2001. El comercio de plantas medicinales: algunos rasgos significativos en el centro de México. In: Rendón, B., S. Rebollar, J. Caballero y M. A. Martínez. (eds.). Plantas, cultura y sociedad. Estudio sobre la relación entre seres humanos y plantas en los albores del siglo XXI. Universidad Autónoma Metropolitana, Unidad Iztapalapa y Secretaría del Medio Ambiente, Recursos Naturales y Pesca. México, D.F., México. pp. 53-75.

Hernández S., L., C. González y F. González. 1991. Plantas útiles de Tamaulipas, México. Anales Inst. Biol. Univ. Nac. Autón. México. Ser. Bot. 62(1): 1-38.

Hernández-Xolocotzi, E. 1970. Exploración etnobotánica y su metodología. Colegio de Postgraduados, Escuela Nacional de Agricultura. Chapingo, México. 43 pp.

Hoffman, B. y T Gallaher. 2007. Importance indices in ethnobotany. Ethnobot. Res. \& Appl. 5: 201-218.

Höft, M., S. K. Barik y A. M. Lykke. 1999. Quantitative ethnobotany. Applications of multivariate and statistical analyses in ethnobotany. People and Plants Working Paper 6. United Nations for Educational, Scientific and Cultural Organization (UNESCO). Paris, Francia. 45 pp.

Lawless H., T. y H. Heymann. 1998. Sensory evaluation of food. Principles and practices. Chapman and Hall. New York, USA. 819 pp.

Luna J., A., L. Montalvo-Espinosa y B. Rendón-Aguilar. 2003. Los usos no leñosos de los encinos en México. Bol. Soc. Bot. Méx. 72: 107-117.

Luna J., A. y B. Rendón. 2008. Recursos vegetales útiles en diez comunidades de la Sierra Madre del Sur, Oaxaca, México. Polibotánica 26: 193-242.

Marín-Corba, C., D. Cárdenas-López y S. Suárez-Suárez. 2005. Utilidad del valor de uso en etnobotánica. Estudio en el Departamento de Putumayo (Colombia). Caldasia 27(1): 89-101.

Martin, G. J. 1995. Etnobotánica, manual de métodos. Pueblos y Plantas 1. Ed. NordanComunidad. Montevideo, Uruguay. 272 pp.

Miranda, F. y E. Hernández X. 1963. Los tipos de vegetación de México y su clasificación. Bol. Soc. Bot. Méx. 28: 29-179.

Monroy-Ortiz, C. y R. Monroy. 2004. Análisis preliminar de la dominancia cultural de las plantas útiles en el estado de Morelos. Bol. Soc. Bot. Méx. 74: 77-95.

Pardo, S. M. y E. Gómez P. 2003. Etnobotánica: aprovechamiento tradicional de plantas y patrimonio cultural. Anales Jard. Bot. Madrid 60(1): 171-182.

Paredes-Flores, M., R. Lira y P. Dávila. 2007. Estudio etnobotánico de Zapotitlán Salinas, Puebla. Acta Bot. Mex. 79: 13-61.

Paule, D. S. y C. Potvin. 2004. Conservation of useful plants: an evaluation of local priorities from two indigenous communities in eastern Panama. Econ. Bot. 58(1): 38-57. 
Pérez-Gil, J. A., S. Chacón M. y R. Moreno R. 2000. Validez de constructo: el uso de análisis factorial exploratorio-confirmatorio para obtener evidencias de validez. Psicothema 12(2): 442-446.

Peters, C. 1996. The ecology and management of non-timber forest resources. Technical Papers 322. World Bank. Washington, D.C., USA. 162 pp.

Rivera, D., C. Obón, C. Inocencio, M. Heinrich, A. Verde, J. Fajardo y J. A. Palazón. 2007. Gathered food plants in the mountains of Castilla-La Mancha (Spain): Ethnobotany and Multivariate Analysis. Econ. Bot. 61(3): 269-289.

Rzedowski, J. 2006. Vegetación de México. Comisión Nacional para el Conocimiento y Uso de la Biodiversidad. 1a. edición digital. México, D.F., México. 504 pp. http:// www.biodiversidad.gob.mx/publicaciones/librosDig/pdf/VegetacionMx_Cont.pdf. Consultada el 8 de diciembre de 2010.

Sánchez, M. J. 1996. Utilización de los recursos naturales y estrategias de reproducción. Estudio de caso en dos comunidades de los valles de Oaxaca. In: Paré, L. y M. J. Sánchez. (coord.). El ropaje de la tierra, naturaleza y cultura en cinco zonas rurales. Plaza y Valdés \& Universidad Nacional Autónoma de México. México, D.F., México. pp. 97-175.

Sharma, S. 1996. Applied multivariate techniques. John Wiley \& Sons, Inc. New York, USA. 493 pp.

Ticktin, T., G. de la Pefia, C. Ilsley, S. Dalle y T. Johns. 2002. Participatory ethnoecological research for conservation: lessons from case studies in Mesoamerica. In: Stepp, J. R., E. S. Wyndham y R. K. Zarger (eds.). Ethnobiology and biocultural diversity: Proceedings of the Seventh International Congress of Ethnobiology. University of Georgia Press. Athens. pp. 575-584.

Toledo, V. M., J. Carabias, C. Toledo y C. González-Pacheco. 1989. La producción rural en México: Alternativas ecológicas. Fundación Universo Veintiuno. México, D.F., México. 402 pp.

Toledo, V. 1994. La diversidad biológica de México. Ciencias 34: 43-59.

Toledo, A. 2003. Ríos, costas, mares, hacia un análisis integrado de las regiones hidrológicas de México. Secretaría de Medio Ambiente y Recursos Naturales, Instituto Nacional de Ecología y El Colegio de Michoacán. México, D.F., México. 117 pp.

Tyler, S. T. 2006. Co-manejo de recursos naturales, aprendizaje local para la reducción de la pobreza. Centro Internacional de Investigaciones para el Desarrollo. Ottawa, Canadá. 112 pp.

Villaseñor, J. L. 2004. Los géneros de las plantas vasculares de la flora de México. Bol. Soc. Bot. Méx. 75: 105-135.

Villaseñor, J. L. y F. Espinosa-García. 2004. The alien flowering plants of México. Divers. Distrib. 10: 113-123. 